\title{
Time to Buffer Overflow in an MMPP Queue
}

\author{
Andrzej Chydzinski \\ Silesian University of Technology \\ Institute of Computer Sciences \\ Akademicka 16, 44-100 Gliwice, Poland \\ Andrzej.Chydzinski@polsl.pl
}

\begin{abstract}
In this report we deal with the time to buffer overflow in a finite-buffer queue with MMPP (Markov-modulated Poisson process) arrivals. The results include a closed-form formula for the transform of the distribution of the time to buffer overflow. The main benefit of this formula is that, using properties of the transform, we can easily compute the average overflow time and all the moments (variance etc). Moreover, by means of an inversion algorithm, we can obtain the probability density function and cumulative distribution function of the overflow time. Analytical results are illustrated by a numerical example based on MMPP parameterization fitted to an IP traffic trace file.
\end{abstract}

Keywords: Performance evaluation, buffer overflow, MMPP queue.

\section{Introduction}

The popularity of MMPP in various areas of network traffic modeling, simulation and performance evaluation is connected with the fact that it is probably the simplest model that allows for precise fitting of the statistical parameters of the traffic, including its autocorrelation structure. In other words, the MMPP, remaining analytically tractable, can reflect burstiness and self-similarity of network traffic which results in reliable performance parameters (like loss ratio, queueing delay, buffer occupancy) obtained using MMPP-based models [1].

In this paper we deal with the time to buffer overflow, an informative performance characteristic for buffering processes in network elements (see [2] for a deeper discussion). As the distribution of the time to overflow heavily depends on the autocorrelation structure of the traffic, it is very important to use a model that is able to mimic that structure properly. MMPP is very well suited for this purpose, especially taking into account that the methodology for fitting traces to MMPP is well developed (see [1],[3]-[6]).

The main result of this paper is a closed-form formula for the Laplace transform of the buffer overflow time distribution in a finite-buffer queue, presented in Theorem 1 Using this formula, we can easily compute the average overflow time, all moments, the probability density function and cumulative distribution function. A finite buffer of size $b$ and general type of service time are assumed, which means that in Kendall's notation we investigate herein the $M M P P / G / 1 / b$ queueing system.

To the best of the author's knowledge there have been no reported results of this type yet. Most of the papers are devoted to other queueing characteristics, mainly to queue 
size distribution or queueing delay (workload). In particular, these characteristics for various MMPP queueing models in the stationary regime can be found by the reader in [7]-[9], while their time-dependent versions in [10]-[15]. There are also some papers in which approximation techniques for analysis of MMPP queues are shown, for instance [16]-[18]. As regards the buffer overflow time, in [19|20] some asymptotic results devoted to related problems can be found. In [21], a solution for discrete-time Markovian queues of $\operatorname{Geom}(n) / \operatorname{Geom}(n) / 1 / N$ type is given.

Finally, in [22] systems with exponential service times are investigated. The approach presented herein is different, and it does not restrict analysis to Markovian or exponential service times, which excludes some cases which are important from a practical point of view, like constant service time. It also has an advantage of giving solutions in a closed, easy to use, form.

The remaining part of paper is organized in the following way. In section 2 , the arrival process, the queueing model and the notation used throughout the article are presented. In section 3, the analysis of the buffer overflow time is performed. In particular, a formal definition of the overflow time is given and the main result of the paper, Theorem 1 is proven. In addition, some computational issues are discussed at the end of that section. In section 4, a numerical illustration based on IP traffic is presented. Finally, remarks concluding the paper are gathered in section 5 .

\section{The Model}

For a very good survey on MMPP we refer the reader to [7]. Following the authors, a Markov-modulated Poisson process can is obtained by varying the arrival rate of the Poisson process according to an $m$-state continuous-time Markov chain. In particular, when the Markov chain is in state $i$, arrivals occur according to a Poisson process of rate $\lambda_{i}$. Therefore MMPP is parametrized by two $m \times m$ matrices:

$Q$ - infinitesimal generator of the continuous-time Markov chain,

$\Lambda=\operatorname{diag}\left(\lambda_{1}, \ldots, \lambda_{m}\right)-$ it has arrival rates on its diagonal, non-diagonal elements are zeros.

In this article we deal with a single-server queue whose arrival process is given by an MMPP. The service time is distributed according to a distribution function $F(\cdot)$, which may assume any form, and the standard independence assumptions are made. The buffer size is finite and equal to $b$, including service position. This means that if a packet (cell, job, customer) at its arrival finds the buffer full, it is blocked and lost.

The following nomenclature is used throughout the paper:

$J(t)$ - the state of the modulating Markov chain at time $t$

$X(t)$ - the queue size at time $t$

$\mathbf{P}(\cdot)$ - the probability

$P_{i, j}(n, t)=\mathbf{P}(N(t)=n, J(t)=j \mid N(0)=0, J(0)=i)-$ the counting function

for the MMPP. $N(t)$ denotes the total number of arrivals in $(0, t]$

$Q_{i j}$-element of the matrix $Q$. This notation is used for all matrices.

$z(s)=\left(\left(s+\lambda_{1}-Q_{11}\right)^{-1}, \ldots,\left(s+\lambda_{m}-Q_{m m}\right)^{-1}\right)^{T}-$ column vector of size $m$ with elements $\left(s+\lambda_{i}-Q_{i i}\right)^{-1}$

$\tilde{d}_{n, i}(s)=\sum_{j=1}^{m} \sum_{k=0}^{n-1} \int_{0}^{\infty} e^{-s t} P_{i, j}(k, t)(1-F(t)) d t$, 


$$
\begin{aligned}
& \tilde{d}_{n}(s)=\left(\tilde{d}_{n, 1}(s), \ldots, \tilde{d}_{n, m}(s)\right)^{T}, \\
& z(s)=\left(\left(s+\lambda_{1}-Q_{11}\right)^{-1}, \ldots,\left(s+\lambda_{m}-Q_{m m}\right)^{-1}\right)^{T} . \\
& a_{k, i, j}(s)=\int_{0}^{\infty} e^{-s t} P_{i, j}(k, t) d F(t), \\
& p_{i j}= \begin{cases}0 & \text { if } i=j, \\
Q_{i j} /\left(\lambda_{i}-Q_{i i}\right) & \text { if } i \neq j .\end{cases}
\end{aligned}
$$

In addition, the following $m \times m$ matrices are used:

$$
\begin{aligned}
\mathbf{0} & =m \times m \text { matrix of zeroes, } \\
I & =m \times m \text { identity matrix, } \\
A_{k}(s) & =\left[a_{k, i, j}(s)\right]_{i, j}, \\
Z(s) & =\left[\frac{\left(\lambda_{i}-Q_{i i}\right) p_{i j}}{s+\lambda_{i}-Q_{i i}}\right]_{i, j}, \\
E(s) & =\left[\frac{\Lambda_{i j}}{s+\lambda_{i}-Q_{i i}}\right]_{i, j}, \\
R_{0}(s) & =\mathbf{0} \\
R_{1}(s) & =A_{0}^{-1}(s), \\
R_{k+1}(s) & =A_{0}^{-1}(s)\left(R_{k}(s)-\sum_{i=0}^{k} A_{i+1}(s) R_{k-i}(s)\right), \quad k \geq 1 .
\end{aligned}
$$

In this notation $\left[b_{i, j}\right]_{i, j}$ denotes an $m \times m$ matrix with elements $b_{i, j}$.

\section{Time to Buffer Overflow}

The time to buffer overflow is denoted herein by $\tau_{n, i}$ and defined formally in the following way. Let $X(t), t \geq 0$ be the queue size process. Let the initial queue size be $n$, $0 \leq n<b$, and the initial state of the modulating Markov process be $i, 1 \leq i \leq m$. Then

$$
\tau_{n, i}=\inf \{t>0: X(t)=b \mid X(0)=n, J(0)=i\} .
$$

Although usually we are interested only in $\tau_{0, i}$, that is in the overflow time starting from empty buffer, the analysis presented below covers all possible initial lengths of the queue.

The distribution of the buffet overflow time will be presented using the transform of the tail of $\tau_{n, i}$, namely:

$$
l_{n, i}(s)=\int_{0}^{\infty} e^{-s t} \mathbf{P}\left(\tau_{n, i}>t\right) d t
$$

and its column vector:

$$
l_{n}(s)=\left(l_{n, 1}(s), l_{n, 2}(s), \ldots, l_{n, m}(s)\right)^{T} .
$$

Using the transform of the tail is very convenient because having calculated $l_{n, i}(s)$ we can easily obtain the expected value of $\tau_{n, i}$ :

$$
\mathbf{E} \tau_{n, i}=l_{n, i}(0),
$$

the transform of its cumulative distribution function: 


$$
\int_{0}^{\infty} e^{-s t} \mathbf{P}\left(\tau_{n, i}<t\right) d t=\frac{1}{s}-l_{n, i}(s),
$$

or the transform of its probability density function:

$$
\int_{0}^{\infty} e^{-s t} \mathbf{P}_{t}^{\prime}\left(\tau_{n, i}<t\right) d t=1-s l_{n, i}(s) .
$$

Theorem 1. For the $M M P P / G / 1 / b$ queue it holds true that:

$$
l_{n}(s)=\sum_{k=0}^{b-n} R_{b-n-k}(s) A_{k}(s) G_{b}^{-1}(s) h_{b}(s)-\sum_{k=1}^{b-n} R_{b-n-k}(s) \tilde{d}_{k}(s), \quad n<b,
$$

where

$$
\begin{gathered}
G_{b}(s)=(I-Z(s)) \sum_{k=0}^{b} R_{b-k}(s) A_{k}(s)-E(s) \sum_{k=0}^{b-1} R_{b-1-k}(s) A_{k}(s), \\
h_{b}(s)=(I-Z(s)) \sum_{k=1}^{b} R_{b-k}(s) \tilde{d}_{k}(s)-E(s) \sum_{k=1}^{b-1} R_{b-1-k}(s) \tilde{d}_{k}(s)-z(s) .
\end{gathered}
$$

Pr o o f of Theorem 1 Conditioning on the first departure epoch we get for $0<n<b$, $1 \leq i \leq m$ :

$$
\begin{aligned}
\mathbf{P}\left(\tau_{n, i}>t\right)= & \sum_{j=1}^{m} \sum_{k=0}^{b-n-1} \int_{0}^{t} \mathbf{P}\left(\tau_{n+k-1, j}>t-u\right) P_{i, j}(k, u) d F(u) \\
& +(1-F(t)) \sum_{j=1}^{m} \sum_{k=0}^{b-n-1} P_{i, j}(k, t),
\end{aligned}
$$

and for $1 \leq i \leq m$ :

$$
\begin{aligned}
\mathbf{P}\left(\tau_{0, i}>t\right)= & \sum_{j=1}^{m} \int_{0}^{t} \mathbf{P}\left(\tau_{0, j}>t-u\right) p_{i j}\left(\lambda_{i}-Q_{i i}\right) e^{-\left(\lambda_{i}-Q_{i i}\right) u} d u \\
& +\sum_{j=1}^{m} \int_{0}^{t} \mathbf{P}\left(\tau_{1, j}>t-u\right) \Lambda_{i j} e^{-\left(\lambda_{i}-Q_{i i}\right) u} d u \\
& +e^{-\left(\lambda_{i}-Q_{i i}\right) t} .
\end{aligned}
$$

The first part of (7) covers the situation where the first departure time $u$ is before $t$ and and the buffer does not get full by the time $u$. The second part covers the situation where the first departure time $u$ is after $t$ and the buffer does not get full by the time $t$. The first part of (8) corresponds to the situation where the modulating state changes by the 
time $t$, while the second part corresponds to the case where the first arrival occurs by the time $t$. Finally, the last part of (8) covers the situation where nothing happens by the time $t$.

Using transforms we obtain for $0<n<b, 1 \leq i \leq m$ :

$$
l_{n, i}(s)=\sum_{j=1}^{m} \sum_{k=0}^{b-n-1} a_{k, i, j}(s) l_{n+k-1, j}(s)+\tilde{d}_{b-n, i}(s)
$$

and for $1 \leq i \leq m$ :

$$
l_{0, i}(s)=\sum_{j=1}^{m} l_{0, j}(s) \frac{p_{i j}\left(\lambda_{i}-Q_{i i}\right)}{s+\lambda_{i}-Q_{i i}}+\sum_{j=1}^{m} l_{1, j}(s) \frac{\Lambda_{i j}}{s+\lambda_{i}-Q_{i i}}+\frac{1}{s+\lambda_{i}-Q_{i i}} .
$$

Using vector notation we have:

$$
\begin{aligned}
l_{n}(s)= & \sum_{k=0}^{b-n-1} A_{k}(s) l_{n+k-1}(s)+\tilde{d}_{b-n}(s), \quad 0<n<b, \\
& l_{0}(s)=Z(s) l_{0}(s)+E(s) l_{1}(s)+z(s) .
\end{aligned}
$$

Replacing $l_{n}(s)=u_{b-n}(s)$ we obtain:

$$
\begin{gathered}
\sum_{k=-1}^{n-1} A_{k+1}(s) u_{n-k}(s)-u_{n}(s)=\psi_{n}(s), \quad 0<n<b, \\
u_{b}(s)=Z(s) u_{b}(s)+E(s) u_{b-1}(s)+z(s),
\end{gathered}
$$

with

$$
\psi_{n}(s)=A_{n}(s) u_{1}(s)-\tilde{d}_{n}(s) .
$$

All possible solutions of the system (9) have the following form:

$$
u_{n}(s)=R_{n}(s) c(s)+\sum_{k=1}^{n} R_{n-k}(s) \psi_{k}(s), \quad n \geq 1,
$$

where $c(s)$ is a vector which does not depend on $n$ (see Theorem 1 in [23]). Putting $n=1$ into 11 we get

$$
c(s)=A_{0}(s) u_{1}(s)
$$

and

$$
u_{n}(s)=\sum_{k=0}^{n} R_{n-k}(s) A_{k}(s) u_{1}(s)-\sum_{k=1}^{n} R_{n-k}(s) \tilde{d}_{k}(s) .
$$

Finally, by means of the boundary condition we get

$$
u_{1}(s)=G_{b}^{-1}(s) h_{b}(s) .
$$

This finishes the proof of Theorem 1 
In order to make (4) useful for practical purposes we have compute first matrices $A_{k}(s), R_{k}(s), Z(s), E(s)$ and vectors $\tilde{d}_{k}(s), z(s)$. Firstly, $Z(s), E(s)$ and $z(s)$ can be computed in an obvious way. Secondly, computing $R_{k}(s)$ is also easy if we know matrices $A_{k}(s)$. Therefore we are reduced to finding an efficient way of calculating $A_{k}(s)$ and $\tilde{d}_{k}(s)$. This can be done by means of the uniformization technique [8]. In particular, for $A_{k}(s)$ we have

$$
A_{n}(s)=\sum_{j=0}^{\infty} \gamma_{j}(s) K_{n, j}
$$

where

$$
\begin{aligned}
K_{0,0} & =I \\
K_{n, 0} & =\mathbf{0}, n \geq 1, \\
K_{0, j+1} & =K_{0, j}\left(I+\theta^{-1} D_{0}\right), \quad \theta=\max _{i}\left\{\left(-D_{0}\right)_{i i}\right\} \\
K_{n, j+1} & =\theta^{-1} \sum_{i=0}^{n-1} K_{i, j} D_{n-i}+K_{n, j}\left(I+\theta^{-1} D_{0}\right), \\
\gamma_{j}(s) & =\int_{0}^{\infty} \frac{e^{-(\theta+s) t}(\theta t)^{j}}{j !} d F(t) .
\end{aligned}
$$

The truncation rule for the sum in (12) can be found in [7]. Vectors $\tilde{d}_{k}(s)$ can be computed using the following $m \times m$ matrices:

$$
\bar{D}_{k}(s)=\left[\int_{0}^{\infty} e^{-s t} P_{i, j}(k, t)(1-F(t)) d t\right]_{i, j} .
$$

The uniformization technique gives now:

$$
\bar{D}_{n}(s)=\sum_{j=0}^{\infty} \delta_{j}(s) K_{n, j}
$$

with

$$
\delta_{j}(s)=\int_{0}^{\infty} \frac{e^{-(\theta+s) t}(\theta t)^{j}}{j !}(1-F(t)) d t .
$$

Naturally, we have

$$
\tilde{d}_{n, i}(s)=\sum_{j=1}^{m} \sum_{k=0}^{n-1}\left(\bar{D}_{k}(s)\right)_{i, j}
$$

Having computed all coefficient matrices and vectors we may proceed to compute the mean buffer overflow time and transforms of its pdf and cdf, using (1), (2) and (3), respectively. If we are also interested in the precise shape of the density function, 
algorithms for the numerical Laplace transform inversion have to be used. For example, in [24] the following method based on the Euler summation formula is proposed:

$$
f(t) \approx \sum_{k=0}^{m} \sum_{j=0}^{n+k}\left(\begin{array}{l}
m \\
k
\end{array}\right) 2^{-m}(-1)^{j} a_{j}(t),
$$

where

$$
\begin{gathered}
a_{k}(t)=\frac{e^{A / 2 t}}{2 l t} \tilde{d}_{k}(t), \quad k \geq 0, \\
\tilde{d}_{0}(t)=f^{*}\left(\frac{A}{2 l t}\right)+2 \sum_{j=1}^{l} \operatorname{Re}\left[f^{*}\left(\frac{A}{2 l t}+\frac{i j \pi}{l t}\right) e^{i j \pi / t}\right], \\
\tilde{d}_{k}(t)=2 \sum_{j=1}^{l} \operatorname{Re}\left[f^{*}\left(\frac{A}{2 l t}+\frac{i j \pi}{l t}+\frac{i k \pi}{t}\right) e^{i j \pi / t}\right], \quad k \geq 1 .
\end{gathered}
$$

$f(t)$ is the original function, $f^{*}(s)$ denotes a transform to be inverted while coefficients $m, n, A, l$ are used to control the inversion error. Typical values are $m=11, n=38$, $A=19$ and $l=1$.

\section{Numerical Illustration}

For numerical purposes, we are going to use a parameterization of MMPP fitted to an IP traffic trace file. Using one million packet headers from the file FRG-11372081981.tsh, recorded at the FGR aggregation point run by PMA (Passive Measurement and Analysis Project [25]), the following MMPP parameteriztion was obtained in [15]:

$$
\begin{gathered}
Q=\left[\begin{array}{rrrrr}
-172.53 & 38.80 & 30.85 & 0.88 & 102.00 \\
16.76-883.26 & 97.52 & 398.9 & 370.08 \\
281.48 & 445.97-1594.49 & 410.98 & 456.06 \\
23.61 & 205.74 & 58.49-598.93 & 311.09 \\
368.48 & 277.28 & 7.91 & 32.45-686.12
\end{array}\right], \\
\left(\lambda_{1}, \cdots, \lambda_{5}\right)=(59620.6,113826.1,7892.6,123563.2,55428.2) .
\end{gathered}
$$

The mean packet size is 850 bytes. Other basic characteristics of the traffic sample and its MMPP model are shown in Table 1. It is important that the autocorrelation function fits the original traffic reasonably well on several time scales (see Fig. 5 in [15]).

It is assumed that service time, $d$, is constant, the initial queue size is 0 , and that the initial state of the modulating process is distributed according to

$$
\pi=(0.52174,0.12808,0.023151,0.11352,0.21351)
$$

which is the steady state vector of the underlying Markov chain, $J(t)$.

Now, manipulating the service time we may obtain different traffic intensities

$$
\rho=d \pi \Lambda 1 \text {. }
$$


Table 1. Parameters of the original and MMPP traffic

\begin{tabular}{|c|c|c|}
\hline & mean interarrival time $[\mu$ s] & arrival rate, $[\mathrm{pkts} / \mathrm{s}]$ \\
\hline original traffic & 13.940 & 71732 \\
\hline MMPP & 13.941 & 71729 \\
\hline
\end{tabular}

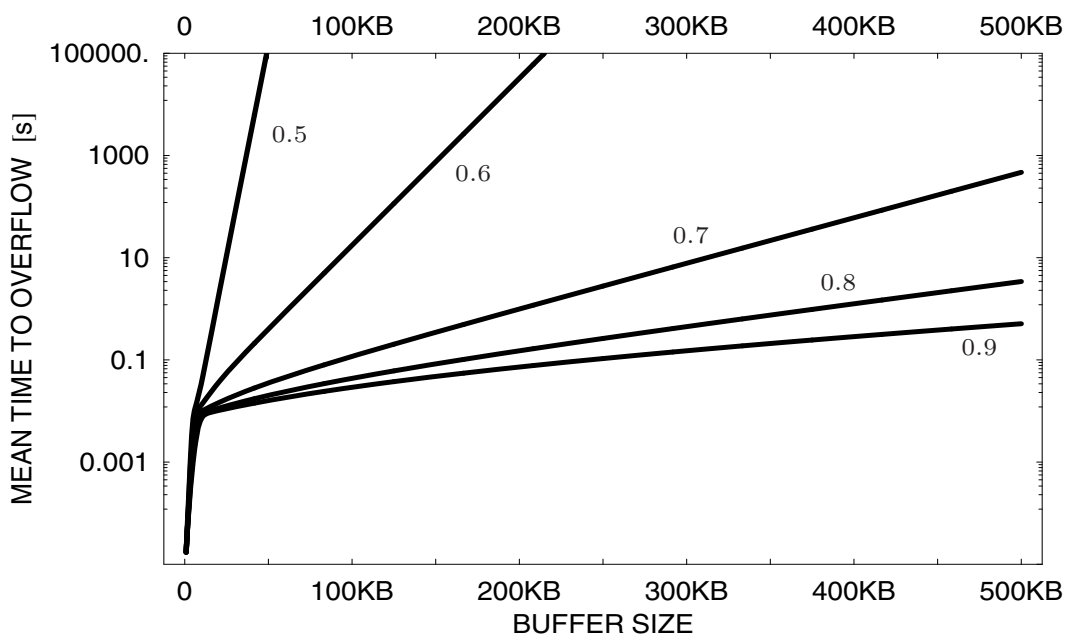

Fig. 1. Mean time to overflow versus the buffer size for MMPP arrivals and five different traffic intensities, namely $0.5,0.6,0.7,0.8$ and 0.9

Sample results for five different traffic intensities are depicted in Figure 1 Each curve represents the average time to overflow as a function of the buffer size in logarithmic scale. Analyzing this set of results we can notice a few things.

Firstly, for large buffers the average overflow time seems to grow linearly (on logscaled plot) with the buffer size. This effect can potentially be used for estimation of the overflow time in large-buffer systems using numerical results obtained for much smaller buffers.

Secondly, a characteristic bend can be observed in a low range (between 5 and 10KB) of each curve. This indicates that for a very small queueing capacity the system's behaviour is significantly different.

Thirdly, the time to overflow decreases when the traffic intensity grows but this effect was to be expected.

Now we demonstrate how the autocorrelation structure of the traffic influences the buffer overflow time. For this purpose we consider the Poisson arrivals instead of MMPP. Naturally, the same arrival rate is assumed. Figure 2 reports average overflow times in this case. As the Poisson process is the simplest case of MMPP, the shapes of curves are similar, but there are great differences in values. For instance, in Table 2 a comparison between MMPP and Poisson-arrival models for $20 \mathrm{~KB}$ of buffering space is shown. The overflow times in the autocorrelated model are always smaller, and the difference grows enormously as the traffic intensity decreases. 


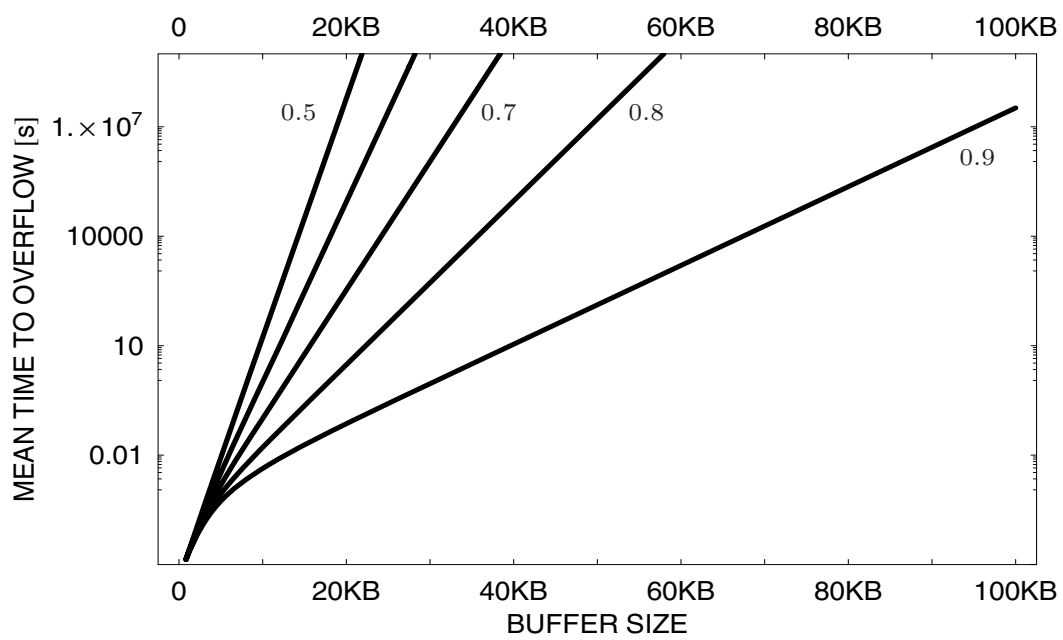

Fig. 2. Mean time to overflow versus the buffer size for Poisson arrivals and five different traffic intensities, namely $0.5,0.6,0.7,0.8$ and 0.9

Table 2. Mean overflow time [s] for MMPP and Poisson arrivals and the buffer size of $20 \mathrm{~KB}$

\begin{tabular}{|c|c|c|}
\hline $\begin{array}{c}\text { traffic } \\
\text { intensity }\end{array}$ & $\begin{array}{c}\text { Poisson } \\
\text { arrivals }\end{array}$ & $\begin{array}{c}\text { MMPP } \\
\text { arrivals }\end{array}$ \\
\hline 0.5 & $5.958 \times 10^{7}$ & $1.629 \times 10^{0}$ \\
\hline 0.6 & $8.780 \times 10^{4}$ & $3.543 \times 10^{-2}$ \\
\hline 0.7 & $3.367 \times 10^{2}$ & $1.441 \times 10^{-2}$ \\
\hline 0.8 & $3.004 \times 10^{0}$ & $1.128 \times 10^{-2}$ \\
\hline 0.9 & $7.256 \times 10^{-2}$ & $9.758 \times 10^{-3}$ \\
\hline
\end{tabular}

\section{Conclusion}

In this paper a comprehensive solution for the buffer overflow time in a finite-buffer queue fed by the Markov-modulated Poisson process is shown. As the MMPP is able to mimic the complex autocorrelation structure of network traffic, this solution may be helpful in the proper sizing of buffers in network elements.

The main result is presented in a closed, easy to use, form. It allows one to obtain all characteristics of the overflow time distribution, including the mean value, the moments, pdf, cdf etc. It has also the following advantage in numerical calculations. As the sequences $A_{k}(s), R_{k}(s), \tilde{d}_{k}(s)$ do not depend on the buffer size, $b$, nor the initial queue length, $n$, after computing these sequences up to some index we can obtain a variety of results for different values of $n$ and $b$ with practically no additional effort.

\section{Acknowledgment}

This work was supported in part by MNiSW under grant N517 025 31/2997. 


\section{References}

1. Salvador, P., Valadas, R. and Pacheco, A. Multiscale Fitting Procedure Using Markov Modulated Poisson Processes. Telecommunication Systems 23:1,2, 123-148, (2003).

2. Schwefel, H.-P., Lipsky, L. and Jobmann, M. On the necessity of transient performance analysis in telecommunication systems, in: Teletraffic Engineering in the Internet Era, eds. J.M. de Souza, N.L.S. da Fonseca and E.A.S. Silva, Elsevier, Amsterdam, (2001).

3. Deng, L. and Mark, J. Parameter estimation for Markov modulated Poisson processes via the EM algorithm with time discretization, Telecommunication Systems 1, pp. 321-338, (1993).

4. Ryden, T. An EM algorithm for parameter estimation in Markov modulated Poisson processes, Comput. Stat. Data Anal. 21, pp. 431447, (1996).

5. Klemm, A., Lindemann, C. and Lohmann, M. Modeling IP traffic using the batch Markovian arrival process. Performance Evaluation, Vol. 54, Issue 2, (2003).

6. Yoshihara, T., Kasahara, S. and Takahashi, Y. Practical time-scale fitting of self-similar traffic with Markov-modulated Poisson process, Telecommunication Systems 17(1/2):185211 (2001).

7. Fischer, W. and Meier-Hellstern, K. The Markov-modulated Poisson process (MMPP) cookbook. Performance Evaluation 18, No.2, 149-171 (1992).

8. Lucantoni, D. M. New results on the single server queue with a batch Markovian arrival process. Commun. Stat., Stochastic Models 7, No.1, 1-46 (1991).

9. Baiocchi, A. and Blefari-Melazzi, N. Steady-state analysis of the MMPP/G/1/K queue. IEEE Trans. Commun. 41, No.4, 531-534 (1992).

10. Lucantoni, D. M., Choudhury, G. L. and Whitt, W. The transient $B M A P / G / 1$ queue. Commun. Stat., Stochastic Models 10, No.1, 145-182 (1994).

11. Le Ny, L.-M. and Sericola, B. Transient Analysis of the $B M A P / P H / 1$ Queue. International Journal of Simulation: Systems, Science \& Technology. Special Issue on Analytical \& Stochastic Modeling Techniques, 3(3-4), (2002).

12. Van Houdt B. and Blondia C. QBDs with marked time epochs: a framework for transient performance measures. Proc. of QEST 2005, Torino, Italy, p. 210-219,(2005).

13. Kulkarni, L. and Li, S.-Q. Transient behaviour of queueing systems with correlated traffic. Perform. Eval. 27\&28, 117-145 (1996).

14. Lee, D.-S. and Li, S.-Q. Transient analysis of multi-server queues with Markov-modulated Poisson arrivals and overload control. Perform. Eval. 16, No.1-3, 49-66 (1992).

15. Chydzinski, A. Transient analysis of the MMPP/G/1/K queue. Telecommunication Systems 32(4): 247-262, (2006).

16. Ross, S. Approximating transition probabilities and mean occupation times in continuoustime Markov chains. Probability in the Engineering and Informational Sciences, 1:251 264, (1987).

17. Carmo, R.M.L.R., de Souza e Silva, E. and Marie, R. Efficient solutions for an approximation technique for the transient analysis of Markovian models. Technical report, IRISA Publication Interne. N 1067, (1996).

18. Van Houdt B. and Blondia C. Approximated transient queue length and waiting time distributions via steady state analysis. Stochastic Models, 21:2/3, p. 725-744, (2005).

19. Glasserman, P. and Kou, S.-G. Limits of first passage times to rare sets in regenerative processes, Ann. Appl. Probab. 5, 424445, (1995).

20. Gnedenko, B. V. and Kovalenko, I. N. Introduction to Queueing Theory, 2nd ed. Birkhuser, Basel, (1989). 
21. Chaudhry, M. L. and Zhao, Y. Q. First-passage time and busy period distributions of discretetime Markovian queues: Geom(n)/Geom(n)/1/N. Queueing Systems, 18: 5-26, (1994).

22. Asmussen, S., Jobmann, M. and Schwefel, H.-P. Exact Buffer Overflow Calculations for Queues via Martingales. Queueing Systems 42(1): 63-90, (2002).

23. Chydzinski, A. The oscillating queue with finite buffer. Performance Evaluation, 57/3 pp. 341-355, (2004).

24. Abate, J. Choudhury, G. L. and Whitt, W. An introduction to numerical transform inversion and its application to probability models. Chapter in Computational Probability, pp. 257-323, W. Grassman (ed.), Kluwer, Boston, 1999.

25. http://pma.nlanr.net/ 